J. Lake Sci. (湖泊科学), 2013, 25(3):392-397

http: //www. jlakes.org. E-mail: jlakes@niglas.ac.cn

(C) 2013 by Journal of Lake Sciences

\title{
基于植物昼夜释氧变化规律的复合垂直流人工湿地氮形态
}

陈梦银 ${ }^{1}$, 朱 伟 ${ }^{1,2}$, 董 婵 ${ }^{2}$

(1: 河海大学环境学院,南京 210098)

(2: 河海大学水资源高效利用与工程安全国家工程研究中心, 南京 210098)

摘 要: 为了研究植物根系释氧规律对人工湿地中硝化与反硝化作用的影响, 采用传统连续运行方式及根据释氧规律调节 的新型运行方式 (白天连续进水、夜间停水), 在复合垂直流人工湿地小试系统中进行实验,采用代表性的挺水植物香蒲作为 湿地植物, 水力负荷为 $0.71 \mathrm{~m}^{3} /\left(\mathrm{m}^{2} \cdot \mathrm{d}\right)$. 结果表明: 系统内白天以好氧的硝化作用为主, 夜间以厌氧、缺氧的反硝化作用为 主, 且在新型运行方式下, 系统内白天亚硝酸盐氮累积量为传统运行方式的 5 倍,硝酸盐氮累积量为其 2 倍, 夜间亚硝酸盐 氮和硝酸盐氮减少量又分别比传统运行方式高 $64 \%$ 和 $26 \%$. 这说明在新型运行方式下, 人工湿地脱氮效果优于传统运行 方式.

关键词: 植物根系释氧规律;香蒲;复合垂直流人工湿地;氮形态;运行方式

\section{Nitrogen forms in integrated vertical-flow on man-made wetland based on the diurnal fluc- tuations of oxygen release in cattail roots}

\section{CHEN Mengyin ${ }^{1}$, ZHU Wei ${ }^{1,2} \&$ DONG Chan ${ }^{2}$}

(1: College of Environment, Hohai University, Nanjing 210098, P. R. China)

(2: National Engineering Research Center of Water Resources Efficient Utilization and Engineering Safety, Hohai University, Nanjing 210098, P. R. China)

Abstract: In order to study the effects of plants' roots oxygen release on the nitrification and de-nitrification in man-made wetlands, we selected two operation modes, namely traditional continuous operation mode and new operation mode (a continuous feeding during the day and stop feeding during the night ). The experiment was carried out in a small integrated vertical-flow man-made wetland system with a hydraulic loading rate of $0.71 \mathrm{~m}^{3} /\left(\mathrm{m}^{2} \cdot \mathrm{d}\right)$, and the representative emergent plant cattail as the wetland plant was chosen. The results showed that the aerobic nitrification took place mainly during the day and the anaerobic-anoxic de-nitrification, during the night, in the system. In addition, the daytime nitrite accumulation was 5 times and nitrate accumulation was 2 times in new operation mode comparing to those in traditional mode, the decrements of nitrite and nitrate were $64 \%$ and $26 \%$ bigger, respectively, than in traditional mode. The new operation mode showed a better nitrogen removal effect.

Keywords: Root oxygen release pattern; cattail; integrated vertical-flow on man-made wetland; nitrogen form; operation mode

人工湿地对污水中有机物和悬浮物的去除效果较好, 然而美中不足之处是一般难以达到良好的脱氮效 果. $V_{y m a z a l}{ }^{[1]}$ 调查了捷克运行 10 年的潜流湿地, 发现有机物和悬浮物的去除率分别达到了 $83.5 \%$ 和 $84.3 \%$, 而总氮 $(\mathrm{TN})$ 和铵态氮 $\left(\mathrm{NH}_{4}^{+}-\mathrm{N}\right)$ 去除率仅为 $41.6 \%$ 和 $42.7 \%$. 在伊朗, 用来处理市政污水的芦苇床 潜流湿地 COD、BOD、总悬浮质的去除率分别达到了 $86 \%$ 、90\% 和 $89 \%$, 而 TN 的去除率只有 $34 \%{ }^{[2]}$. 宋新山

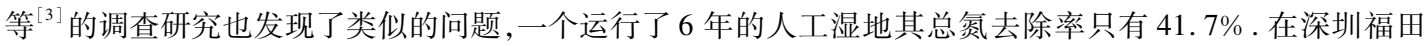
区,用于处理市政污水的某人工湿地其 TN 和铵态氮的去除率分别为 $46 \%$ 和 $50 \%{ }^{[4]}$, 因此人工湿地较差的 脱氮效果使其应用受到一定限制.

* 国家重点基础研究发展计划“973”项目(2012CB719804)、江苏省重点基金项目 (BK2011025)、国家自然科学基金 项目 (50979028) 和中国博士后科学基金项目 (2012 M511675) 联合资助. 2012 - 09-11 收稿;2012 - 12 - 19 收修改 稿. 陈梦银,女, 1988 年生, 硕士研究生;E-mail: zhuweiteam. hhu@ gmail. com. 
人工湿地中氮的去除以微生物的硝化与反硝化反应为主 ${ }^{[5-7]}$, 这一过程的实现需要营造一个好氧一庈氧 相结合的环境, 因此人工湿地中的溶解氧 (DO) 浓度成为影响该过程的主要因素之一 ${ }^{[8]}$. Zhang 等 $^{[8]}$ 在其研 究中通过人工微曝气提高湿地中的 DO 水平,使 TN 去除率达到了 $86 \%$, 比没有曝气的湿地提高 $15 \%$. Fan 等 ${ }^{[9]}$ 在其研究中发现, 连续曝气人工湿地虽有很高的铵态氮转化率但 TN 去除率仅为 $29 \%$, 而间歇曝气人工 湿地 TN 去除率为 $74 \%$. 一般连续运行的潜流人工湿地难以在时间和空间上实现明显的好氧一厌氧分段过 程 ${ }^{[10]}$, 这就影响了湿地的脱氮效果.

对脱氮而言, 最理想的环境就是好氧和厌氧条件兼备, 在湿地中实现好氧一厌氧过程的依次发生对脱氮 会具有良好的促进作用. Dong 等 ${ }^{[11]}$ 的研究发现湿地中植物根系释氧存在随光强变化而变化的规律, 白天释 氧、夜间停止释氧, 变化规律符合高斯函数的单峰曲线. 从植物释氧的规律来看, 湿地中存在白天 DO 浓度 高,夜间 DO 浓度低的变化过程.

基于湿地植物这一自然释氧规律, 本文使用复合垂直流人工湿地单元, 根据植物释氧规律调节不同的 运行方式,研究人工湿地中植物释氧的昼夜变化对硝化与反硝化反应所产生的影响.

\section{1 材料与方法}

\section{1 实验污水}

本实验自配污水中氮形态基本采用 $\mathrm{NH}_{4}^{+}-\mathrm{N}$, 主要目的是了解根系释氧变化对 $\mathrm{NH}_{4}^{+}-\mathrm{N}$ 经历硝化反应, 其 后产物硝态氮 $\left(\mathrm{NO}_{3}^{-}-\mathrm{N}\right)$ 又经历反硝化反应的一个完整过程所产生的影响. TN 用硫酸铵配制, COD 用葡萄糖 配制, 总磷 ( TP ) 用磷酸二氢钾配制. 进水的 $\mathrm{COD} 、 \mathrm{DO} 、 \mathrm{TN} 、 \mathrm{NH}_{4}^{+}-\mathrm{N} 、 \mathrm{NO}_{3}^{-}-\mathrm{N}$ 、亚硝态氮 $\left(\mathrm{NO}_{2}^{-}-\mathrm{N}\right)$ 和 $\mathrm{TP}$ 含量分 别为 $115.33 \pm 0.61 、 1.60 \pm 0.20 、 15.34 \pm 1.15 、 14.47 \pm 0.52 、 0.81 \pm 0.12 、 0.02 \pm 0.01$ 和 $0.32 \pm$ $0.05 \mathrm{mg} / \mathrm{L}$.

\section{2 复合垂直流人工湿地实验装置}

1.2. 实验模型 为营造好氧一厌氧交替的环境, 本实验采用复合垂直流人工湿地系统, 实验装置见图 1. 有 机玻璃槽尺寸为 $0.30 \mathrm{~m}$ (长) $\times 0.15 \mathrm{~m}$ (宽) $\times 1.00 \mathrm{~m}$ (高), 由下行池和上行池组成, 两池中间设有隔板 (隔 板高 $0.75 \mathrm{~m}$ ), 底部相通. 两池内均种植香蒲 (种植密度约 100 株 $/ \mathrm{m}^{2}$ ), 填料选用粒径为 $2 \sim 5 \mathrm{~mm}$ 的火山渣, 填料高 $0.75 \mathrm{~m}$, 孔隙率为 0.47 . 污水从下行池顶部流人, 沿程流动, 从上行池顶部流出.

1.2 .2 运行参数 ( A 组、B 组) 小试系统前期经过 5 个月稳定运行, 水力负荷为 $0.71 \mathrm{~m}^{3} /\left(\mathrm{m}^{2} \cdot \mathrm{d}\right)($ 经过一 个昼夜初始进水刚好排出系统). 为研究植物释氧的 昼夜变化对硝化一反硝化作用的影响,本实验根据植 物释氧时段采用两组运行方式, $\mathrm{A}$ 组昼夜连续进水 (以下简称连续运行) ; B 组日间连续进水 ( $7: 00-$ $17: 00$,植物释氧时段), 夜间停水 $(17: 00-5: 00$, 植物 无释氧时段) (以下简称间歇运行).

1.2 .3 采样监测方法 沿程在根系区及非根系区选 取 5 个采样点, 编号分别为 $1^{\#} 、 2^{\#} 、 3^{\#} 、 4^{\#} 、 5^{\#}$ (图 1), 其 中 $1^{\# 、} 、 4^{\#}$ 取样口距填料表面 $30 \mathrm{~cm}, 2^{\#} 、 3^{\#}$ 取样口距填 料表面 $60 \mathrm{~cm}, 5^{\#}$ 为出水口. 每个实验周期为 $22 \mathrm{~h}$, 在沿 程各取样口采样分析, 取样时间为 $7: 00 、 9: 00 、 11: 00$ 、 $13: 00 、 15: 00 、 17: 00 、 20: 00 、 23: 00 、 2: 00 、 5: 00$. 测定水 质指标为 $\mathrm{DO} 、 \mathrm{TN} 、 \mathrm{NH}_{4}^{+}-\mathrm{N} 、 \mathrm{NO}_{3}^{-}-\mathrm{N} 、 \mathrm{NO}_{2}^{-}-\mathrm{N}$, 各指标的测 定方法参照《水和废水监测分析方法》(第四版 $)^{[12]}$.

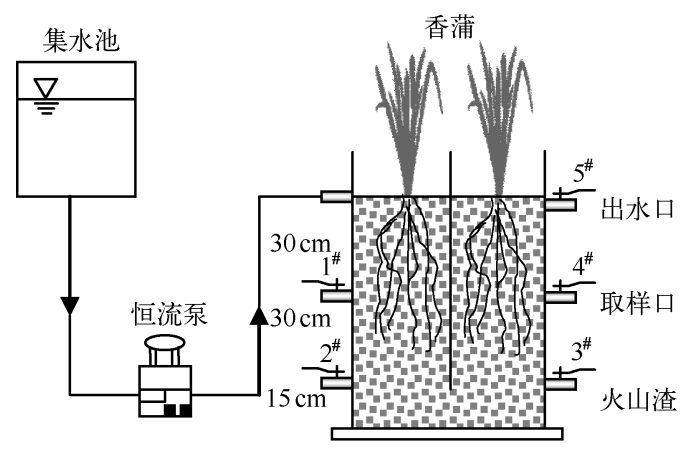

图 1 复合垂直流人工湿地小试系统示意

Fig. 1 Schematic diagram of the integrated vertical-flow on man-made wetland

\section{2 结果}

\section{1 溶解氧昼夜变化}

DO 的监测从当日早上 7:00 点开始至次日早上 $5: 00$ 点结束, 历经 $22 \mathrm{~h}$, 同时也实测了当日光强变化 
(图中 PAR 为光强). 在董婵 ${ }^{[13]}$ 的研究中发现, 植物根系释氧一般会滞后于光强 $2 \mathrm{~h}$ 并出现一个高斯曲线分 布 (图 2), 在夜间释氧基本停止.

从 DO 的变化规律来看,两种运行方式中 DO 浓度都出现了白天高、夜间低的过程. 从沿程变化来看,无 论是下行池还是上行池, 都是表层 ( $1^{\#} 、 4^{\#}$ 测点) 的 DO 浓度高, DO 在 13:00-15:00 出现峰值, 可达 $5 \mathrm{mg} / \mathrm{L}$, 而底层 ( $2^{\#} 、 3^{\#}$ 测点) DO 浓度较低,昼夜间变化也较小.

将两种运行方式下湿地中 DO 值进行比较, 可以看出间歇运行方式的 DO 值在各个深度上都比昼夜连 续运行方式的大. 对间歇运行方式而言, 除下行池表层 ( $1^{\#}$ 测点) 和出口 ( $5^{\#}$ 测点) 在白天出现明显的峰值变 化外, 其他测点的 DO 相对稳定在一个水平上. 而昼夜连续运行方式不论是哪一个测点, 白天都出现明显上 升趋势, 体现出变动的特点. 夜间从 20:00 以后, 间歇运行方式下的 DO 值不论哪个测点都降到 $1 \mathrm{mg} / \mathrm{L}$ 以 下, 底层减少到 $0.5 \mathrm{mg} / \mathrm{L}$ 以下. 从变化量来看表层降低到白天的 $1 / 4$ 以下, 底层降低到白天的 $1 / 3$ 以下, 夜 间溶氧降低明显. 而昼夜连续运行方式下的 DO 在夜间也降低, 大多在 $1 \sim 2 \mathrm{mg} / \mathrm{L}$ 之间, 除下行池表层 ( $1^{\#}$ 测 点) 昼夜间 DO 变化比较显著以外, 其他部位 DO 变化相对不明显. 可以认为, 就两种运行方式而言, 间歇运 行方式整体呈现出白天氧多、夜间氧少、昼夜间 DO 值变化大的特点.

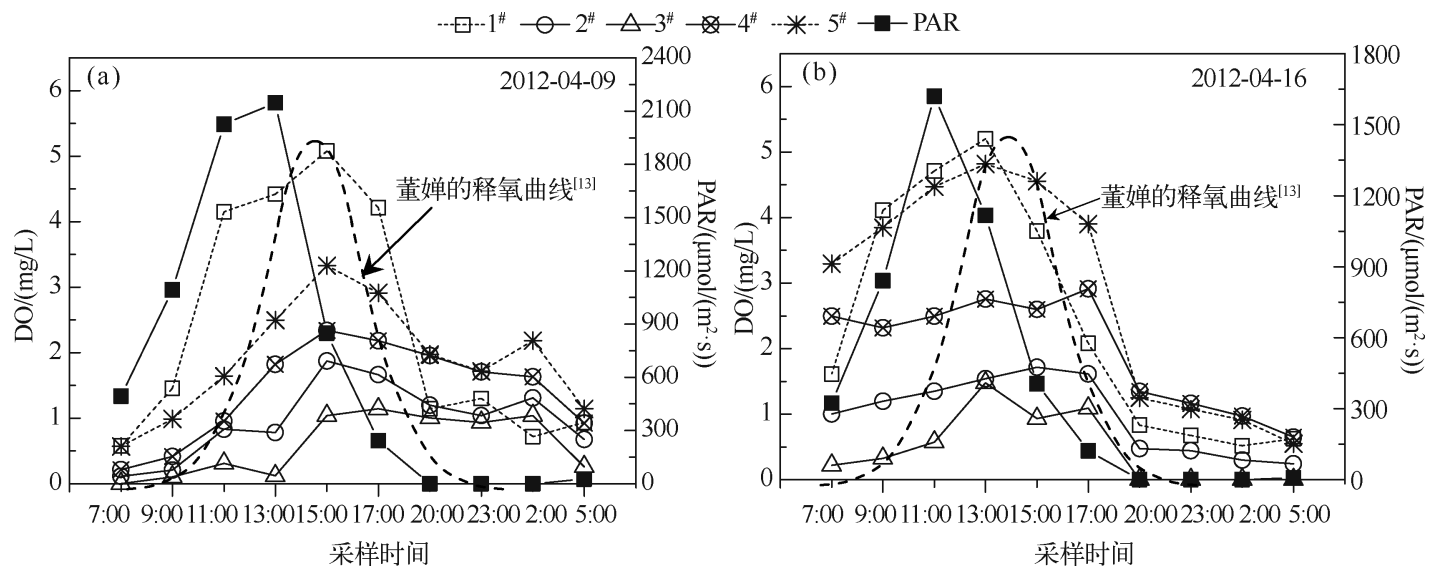

图 2 连续运行方式 (a) 和间歇运行方式 (b)下沿程各点 DO 浓度昼夜变化

Fig. 2 Diurnal fluctuation of DO concentration of all the samples under continuous operation mode(a) and intermittent operation mode(b)

\section{$2.2 \mathrm{NH}_{4}^{+}-\mathrm{N}$ 昼夜变化}

为了解 $\mathrm{DO}$ 变化对各形态氮的影响, 以硝化反应的主体 $\mathrm{NH}_{4}^{+}-\mathrm{N}$ 为对象,整理了数据的变化规律 (图 3 ). 连续运行方式下, $1^{\#} 、 2^{\#}$ 与 $5^{\#}$ 测点在白天 $7: 00-17: 00$ 溶氧较高时段, $\mathrm{NH}_{4}^{+}-\mathrm{N}$ 浓度随时间逐渐降低, 而在夜 间 $17: 00-20: 00$ 低溶氧时段 $\mathrm{NH}_{4}^{+}-\mathrm{N}$ 浓度随时间又慢慢回升. $3^{\#}$ 测点则在一个昼夜内 $\mathrm{NH}_{4}^{+}-\mathrm{N}$ 浓度逐渐上 升, $4^{\#}$ 测点浓度在经历短暂的上升后, 自 $13: 00$ 又开始下降,夜间又略微回升(图 3a).

间歇运行方式下,整体来看各测点 $\mathrm{NH}_{4}^{+}-\mathrm{N}$ 变化规律与连续运行方式下类似,而由于该运行方式下水流 流速变大, 单位时间内进水 $\mathrm{NH}_{4}^{+}-\mathrm{N}$ 量大于连续运行方式, 各测点在白天 $\mathrm{NH}_{4}^{+}-\mathrm{N}$ 浓度值与连续运行方式的 相比都较大,而夜间波动则较小 ( 图 3b).

\section{$2.3 \mathrm{NO}_{2}^{-}-\mathrm{N}$ 昼夜变化}

连续运行方式下, $1^{\#}$ 与 $5^{\#}$ 测点 $\mathrm{NO}_{2}^{-}-\mathrm{N}$ 在 7:00-20:00 间有一个明显累积的过程, 白天浓度明显上升, 其 峰值出现在 $15: 00$, 与 DO 峰值出现时间一致, 其后浓度降低, 在夜间 $17: 00-5: 00$ 间, $1^{\#}$ 与 $5^{\#}$ 测点 $\mathrm{NO}_{2}^{-}-\mathrm{N}^{-}$降 低了约 $55 \% ; 2^{\#} 、 3^{\#}$ 与 $4^{\#}$ 测点与其余两个测点相比, 在一个昼夜内波动不大 (图 $4 \mathrm{a}$ ).

间歇运行方式下, $1^{\#}$ 取样点与其他取样点 $\mathrm{NO}_{2}^{-}-\mathrm{N}$ 浓度变化明显不同, 在 7:00-20:00 间浓度先上升后 下降, 最大值出现在 $15: 00$, 与 DO 最大值出现时间接近, 17:00 停止进水后浓度迅速降低, 至 20:00 降低了 $90 \%$, 比连续运行方式下的降幅大 $64 \%$; 其余 4 个取样点一个周期内基本无变化,波动很小 (图 4b). 
$\cdots-^{\#}-\bigcirc-2^{\#} \triangle 3^{\#}-4^{\#} \cdots *-5^{\#}$
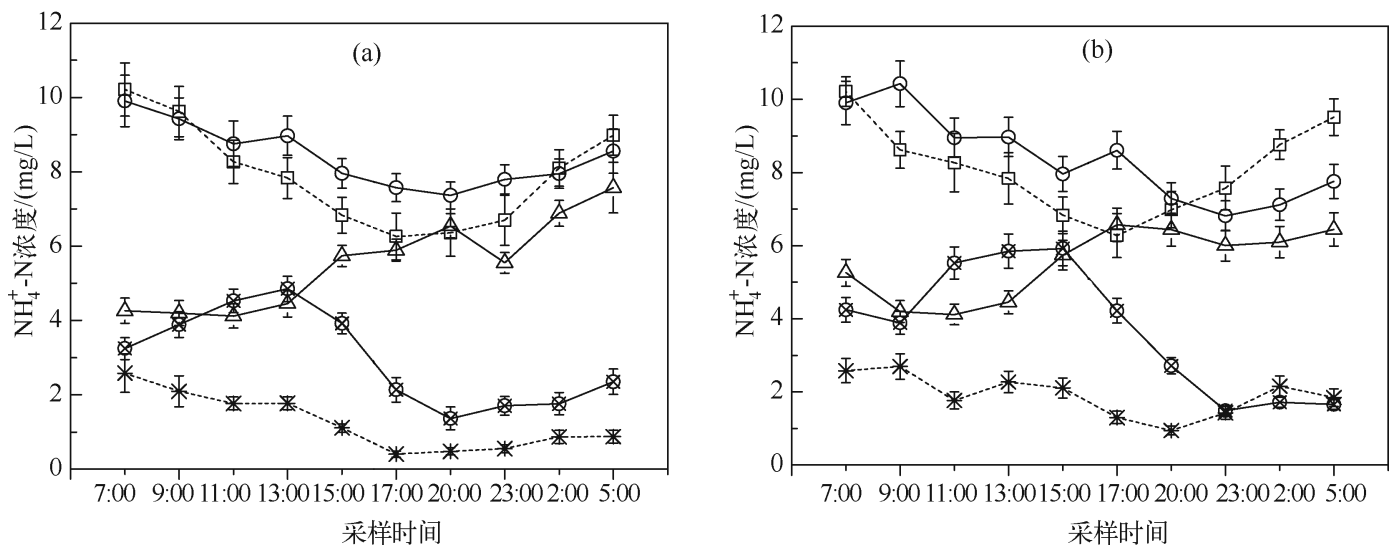

图 3 连续运行方式 (a) 和间歇运行方式 (b) 下沿程各点 $\mathrm{NH}_{4}^{+}-\mathrm{N}$ 浓度昼夜变化

Fig. 3 Diurnal fluctuation of $\mathrm{NH}_{4}^{+}-\mathrm{N}$ concentration of all the samples under continuous operation mode(a) and intermittent operation mode(b)

$\cdots \boxminus-1^{\#} \bigcirc 2^{\#} \triangle 3^{\#}-4^{\#} \cdots *-5^{\#}$
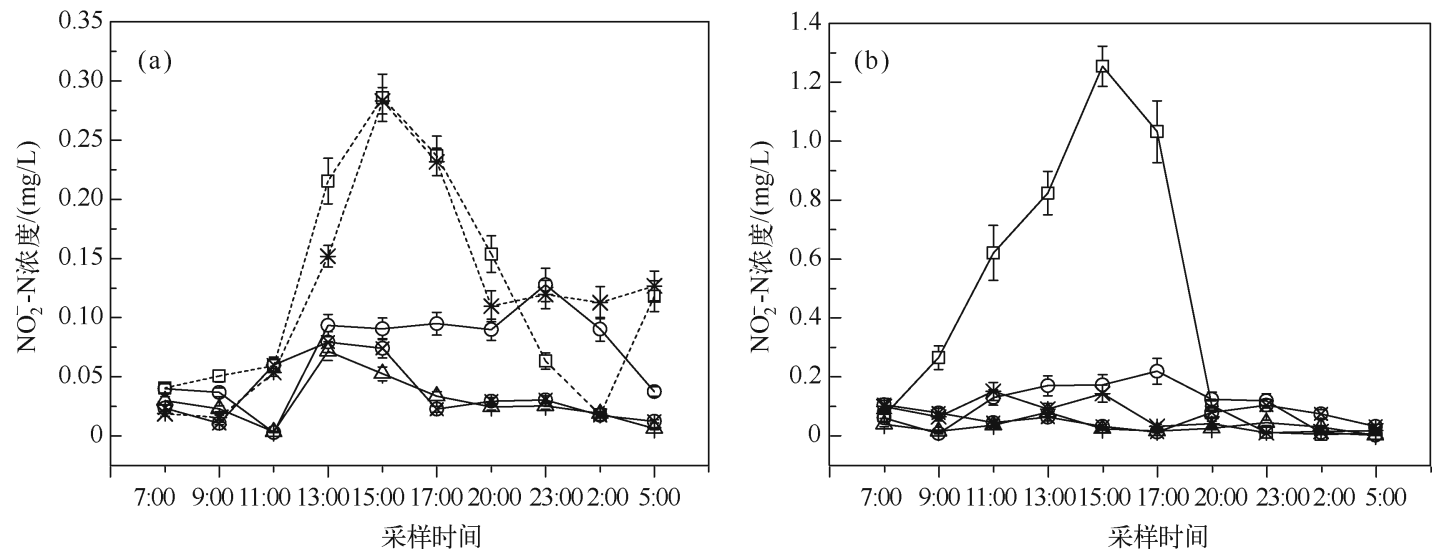

图 4 连续运行方式 (a) 和间歇运行方式 (b) 下沿程各点 $\mathrm{NO}_{2}^{-}-\mathrm{N}$ 浓度昼夜变化

Fig. 4 Diurnal fluctuation of $\mathrm{NO}_{2}^{-}-\mathrm{N}$ concentration of all the samples under continuous operation mode(a) and intermittent operation mode(b)

与连续运行方式相比, 间歇运行方式下各测点 $\mathrm{NO}_{2}^{-}-\mathrm{N}$ 浓度均较高, 峰值浓度为其 4 倍, 且夜间浓度降幅 也较大.

\section{2. $4 \mathrm{NO}_{3}^{-}-\mathrm{N}$ 昼夜变化}

连续运行方式下, $5^{\#}$ 测点在白天 7:00-17:00 $\mathrm{NO}_{3}^{-}-\mathrm{N}$ 浓度逐渐上升, 达到峰值后开始下降, 其余 4 个测 点 $\mathrm{NO}_{3}^{-}-\mathrm{N}$ 浓度在 7:00-9:00 间略有降低, 而后又逐渐升高, 至 20:00 达到最大值后又逐渐下降. 夜间 $20: 00$ 至 $5: 00$, 系统整体 $\mathrm{NO}_{3}^{-}-\mathrm{N}$ 浓度降低约 50\% (图 5a).

间歇运行方式下, $1^{\#}$ 与 $5^{\#}$ 测点从 7:00 至 17:00 $\mathrm{NO}_{3}^{-}-\mathrm{N}$ 浓度逐渐上升, 17:00 达到峰值后开始下降, 其余 取样点在 $7: 00-20: 00$ 间 $\mathrm{NO}_{3}^{-}-\mathrm{N}$ 浓度均逐渐上升,至 $20: 00$ 达到最大值后又逐渐下降,夜间 $20: 00$ 至 $5: 00$, 系统整体 $\mathrm{NO}_{3}^{-}-\mathrm{N}$ 浓度降低约 63\%（图 5b).

对比 2 种运行方式发现, 间歇运行方式下白天系统内 $\mathrm{NO}_{3}^{-}-\mathrm{N}$ 浓度比连续运行方式下的大, 峰值浓度为 其 1.37 倍, $\mathrm{NO}_{3}^{-}-\mathrm{N}$ 浓度降幅也比连续运行方式下的高 $26 \%$. 

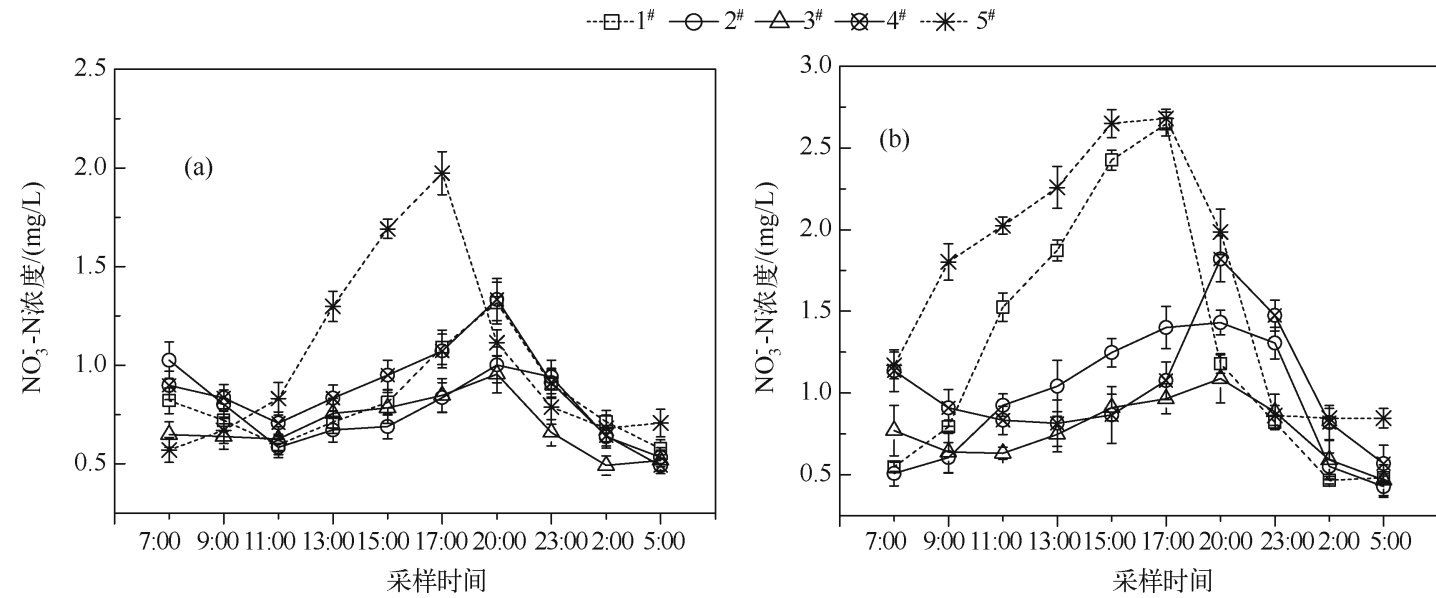

图 5 连续运行方式 (a) 和间歇运行方式 (b) 下沿程各点 $\mathrm{NO}_{3}^{-}-\mathrm{N}$ 浓度昼夜变化

Fig. 5 Diurnal fluctuation of $\mathrm{NO}_{3}^{-}-\mathrm{N}$ concentration of all the samples under continuous operation mode(a) and intermittent operation mode(b)

\section{3 讨论}

白天, 湿地中植物进行光合作用产生的氧气, 除释放到空气中外, 剩余部分通过根系释放到根围基质环 境中, 且随着光强的增大, 根系释氧速率也变大 ${ }^{[13]}$, 这使得白天系统内溶解氧浓度较高, 尤其是根系区 ${ }^{[14]}$, 硝化细菌的活性变大. 在 $\mathrm{DO}$ 充足的条件下, 进水中 $\mathrm{NH}_{4}^{+}-\mathrm{N}$ 被亚硝化菌转化为 $\mathrm{NO}_{2}^{-}-\mathrm{N}$ 后, 进一步被硝化菌 转化为 $\mathrm{NO}_{3}^{-}-\mathrm{N}$. 从实验结果来看, 白天下行池 $\left(1^{\#} 、 2^{\#}\right)$ 及上行池表层 $\left(5^{\#}\right) \mathrm{NH}_{4}^{+}-\mathrm{N}$ 浓度随时间逐渐降低, $1^{\#} 、 5^{\#}$ 测点 $\mathrm{NO}_{3}^{-}-\mathrm{N}$ 浓度则随时间而升高且有一个明显的累积过程, 说明在系统表层发生了明显的亚硝化与硝化 反应 ${ }^{[15]}, \mathrm{NH}_{4}^{+}-\mathrm{N}$ 单位时间转化速率随时间变大. $3^{\# 、} 、 4^{\#}$ 测点处于上行池底部和中部, 相对于下行池及上行池 表层, $\mathrm{DO}$ 浓度较低, $\mathrm{NH}_{4}^{+}-\mathrm{N}$ 转化速率相对较慢, 故 $3^{\# 、} 4^{\#}$ 测点 $\mathrm{NH}_{4}^{+}-\mathrm{N}$ 随时间有所累积, 这与吴振斌、张迎颖 等的研究结果一致 ${ }^{[16-17]}, \mathrm{NO}_{3}^{-}-\mathrm{N}$ 浓度随时间缓慢升高,但相对于表层,升幅较小.

夜间, 植物停止光合作用, 根系无释氧, 且植物自身呼吸作用消耗基质环境中的 DO, 这就使系统内部的 DO 浓度大幅降低, $\mathrm{NH}_{4}^{+}-\mathrm{N}$ 的转化速率较白天变慢,各测点在夜间 $\mathrm{NH}_{4}^{+}-\mathrm{N}$ 浓度随时间慢慢回升; 而低溶氧环 境为反硝化菌创造了良好的条件, 系统内 $\mathrm{NO}_{3}^{-}-\mathrm{N}$ 在反硝化菌的作用下被转化为氮气, $\mathrm{NO}_{3}^{-}-\mathrm{N}$ 的浓度在夜间 大幅降低.

对于连续运行方式来说, 在夜间, 忽略大气复氧的情况,进水携氧仍然是系统的氧源, 系统内整体 DO 水 平维持在 $1 \sim 2 \mathrm{mg} / \mathrm{L}$, 这对于反硝化作用来说并不是最有利的条件. 改变人工湿地运行方式后, 在白天, 与连 续运行方式相比, 流速的增大使得单位时间内系统中氧的传输速率变大, 系统内各部位 DO 都比传统连续运 行方式下高, 且硝化反应的底物 $\mathrm{NH}_{4}^{+}-\mathrm{N}$ 随进水也增多, 这就使好氧的硝化作用变强 ${ }^{[18]}$, 从产物 $\mathrm{NO}_{3}^{-}-\mathrm{N}^{\text {来 }}$ 看, 新的运行方式下 $\mathrm{NO}_{3}^{-}-\mathrm{N}$ 的累积量明显变大, 是传统连续运行方式的 5 倍. 而在夜间, 系统停止进水, 系统 内没有任何外部氧源, 外加植物呼吸作用消耗掉一部分 DO, 使得整个系统内的 DO 水平很低 $(<1 \mathrm{mg} / \mathrm{L})$, 呈 现厌氧缺氧环境, 相对于传统运行方式, 新的运行方式下系统内的环境更有利于反硝化作用的进行, $\mathrm{NO}_{3}^{-}-\mathrm{N}$ 浓度的降幅比传统运行方式下平均大 $45 \%$.

白天进水、夜间停水的运行方式很好地利用了植物根系释氧这一客观规律, 白天根系释氧作为氧源为 系统提供 DO, 有利于好氧的硝化作用, 夜间植物又作为 DO 的消耗体消耗系统内的 DO, 有利于厌氧缺氧的 反硝化作用, 故基于硝化与反硝化作用的氧浓度差, 这种运行方式为硝化与反硝化作用的进行营造了更有 利的环境.

本研究为实际人工湿地工程的运行提供了一种新思路,新型运行方式在不增加额外运行费用的基础上 充分利用湿地植物自身的释氧规律, 根据进水与停水时间的比例调节进水流速来达到与传统运行方式相同 
的水力负荷, 最终达到优化脱氮的目的, 对人工湿地处理分散型农村生活污水有一定的参考价值.

\section{4 结论}

1) 在本实验中, 无论是传统连续运行方式还是新型运行方式 (白天连续进水、夜间停水), 湿地中 DO 都 呈现出白天含量高, 夜间含量低的规律. 并且在新型运行方式下, 系统内 DO 含量在白天比连续运行方式下 高 $45 \%$,在夜间又比连续运行方式下低 $60 \%$,昼夜变化相对更大.

2 ) 在复合垂直流人工湿地小试系统中, 探讨了植物根系释氧的昼夜变化对系统内硝化与反硝化作用的 影响. 在白天, 由于植物根系释氧的补给, DO 较为充足, 系统内以好氧的硝化作用为主; 夜间植物无释氧, 且 呼吸作用消耗 DO, 系统内以厌氧一缺氧的反硝化作用为主. 并且在新型运行方式下, 系统内硝化与反硝化作用 强度得到了提高, 白天 $\mathrm{NO}_{2}^{-}-\mathrm{N}$ 累积量为传统运行方式的 5 倍, $\mathrm{NO}_{3}^{-}-\mathrm{N}$ 累积量为其 2 倍, 夜间 $\mathrm{NO}_{2}^{-}-\mathrm{N}$ 和 $\mathrm{NO}_{3}^{-}-\mathrm{N}$ 减少量又分别比传统运行方式大 $64 \%$ 和 $26 \%$.

\section{5 参考文献}

[ 1 ] Vymazal J. The use of sub-surface constructed wetlands for wastewater treatment in the Czech Republic: 10 years experience. Ecological Engineering, 2002 ,18(5) :633-646.

[ 2 ] Kivaisi AK. The potential for constructed wetlands for wastewater treatment and reuse in developing countries: a review. Ecological Engineering, $2001,16: 545-560$.

[3] 宋新山, 邓 伟, 夏永云. 潜流构建湿地氮素转化运移的理论模型研究. 地球科学进展,2007,22(10):1041-1047.

[ 4 ] Yang Q, Tam NFY, Wong YS et al. Potential use of mangroves as constructed wetland for municipal sewage treatment in Futian, Shenzhen, China. Marine Pollution Bulletin, 2008,57(6-12):735-743.

[5] 李科德,胡正嘉. 芦苇床系统净化污水的机理. 中国环境科学, 1995,15(2):140-144

[6] 贺 锋, 吴振斌, 付贵萍等. 复合构建湿地运行初期理化性质及氮的变化. 长江流域资源与环境, 2002, 11 (3): 279-283.

[ 7 ] Vymazal J. Long-term performance of constructed wetlands with horizontal sub-surface flow: Ten case studies from the Czech Republic. Ecological Engineering, 2011,37(1):54-63.

[ 8 ] Zhang LY, Zhang L, Liu YD et al. Effect of limited artifcial aeration on constructed wetland treatment of domestic wastewater. Desalination, 2010,250 (3) $: 915-920$.

[ 9 ] Fan JL, Liang S, Zhang B et al. Enhanced organics and nitrogen removal in batch-operated vertical flow constructed wetlands by combination of intermittent aeration and step feeding strategy. Environmental Science and Pollution Research (online).

[10] Vymazal J. Removal of nutrients in various types of constructed wetlands. Science of the Total Environment, 2007,380(1/ $2 / 3): 48-65$.

[11] Dong C, Zhu W, Gao M et al. Diurnal fluctuations in oxygen release from roots of Acorus calamus Linn. in a modeled constructed wetland. Journal of Environmental Science and Health Part A, 2011 ,46 :224-229.

[12] 国家环境保护总局《水和废水监测分析方法》编委会. 水和废水监测分析方法:第 4 版. 北京: 中国环境科学出版 社, 2002 .

[13] 董 婵. 人工湿地植物释氧规律及对污染物去除的影响 [学位论文]. 南京: 河海大学,2011.

[14] 黄丹萍, 贺 锋, 吴振斌等. 高氮磷胁迫下菖蒲 (Acorus calamus Linn.) 通气组织和根系释氧的响应. 湖泊科学, $2012, \mathbf{2 4}$ ( 1 ) :83-88.

[15] Zhang LY, Xia XF, Zhao Y et al. The ammonium nitrogen oxidation process in horizontal subsurface flow constructed wetlands. Ecological Engineering, 2011,37(11):1614-1619.

[16] 吴振斌,徐光来, 周培疆等. 复合垂直流人工湿地污水氮的去除效果研究. 农业环境科学学报, 2004, 23 (4): 757-760.

[17] 张迎䓉, 丁为民, 陈秀娟等. 复合垂直流人工湿地的脱氮机理及影响因素分析. 环境工程,2009,27(5):36-40.

[18] 刘 明, 黄 否, 高 旭等. 潜流湿地中微生物对三峡库区微污染水净化效果的影响. 湖泊科学, 2012, 24 (5): 687-692. 